\title{
Reproducibility of Serial Left Ventricle Perfusion, Volume, and Ejection Fraction Measurements Using Multiplexed Multipinhole SPECT in Healthy Rats and Rats After Myocardial Infarction
}

\author{
Jared H. Strydhorst ${ }^{1,2}$, Frans H. Leenen ${ }^{1}$, Terrence D. Ruddy ${ }^{1}$, and R. Glenn Wells ${ }^{1,2}$ \\ ${ }^{I}$ Division of Cardiology, University of Ottawa Heart Institute, Ottawa, Ontario, Canada; and ${ }^{2}$ Department of Physics, Carleton \\ University, Ottawa, Ontario, Canada
}

\begin{abstract}
Assessment of small-animal cardiac data acquired using SPECT requires an accurate understanding of the reproducibility and the uncertainties associated with the technique. Furthermore, it is also useful to have a baseline of reference data against which to compare the outcome of a particular study. Methods: We scanned both healthy and post-myocardial infarction rats injected with ${ }^{99 m} \mathrm{Tc}$-tetrofosmin in a multidetector, multipinhole small-animal SPECT scanner. In this paper, we report on the creation of a reference database of the relative myocardial blood perfusion of rats. We also evaluated the reproducibility of perfusion measurements and measurements of left ventricle volume and ejection fraction, defined as the SD of a particular measurement repeated on the same animal or over multiple animals. Results: For the healthy rats, interscan reproducibility of volume measurements was $4 \%-7 \%$ of the total volume, and intersubject reproducibility was $9 \%-12 \%$ of the volume being measured. Interscan reproducibility remained unaffected after infarction (6\%-8\%), but intersubject reproducibility was much poorer (15\%-26\%). Ejection fraction in healthy animals was highly reproducible between scans and between rats: $3.1 \%$ and $3.3 \%$, respectively. Interscan reproducibility of the postinfarction ejection fraction was $3.6 \%$; intersubject reproducibility after infarction was $8.1 \%$. Conclusion: We have created a reference database for small-animal SPECT perfusion measurements in healthy male Sprague-Dawley rats and quantified the reproducibility of perfusion and functional measurement made with small-animal SPECT in healthy and postinfarction rats.
\end{abstract}

Key Words: rats; multiplexed multipinhole SPECT; gated SPECT; myocardial perfusion; ventricular function

J Nucl Med 2011; 52:1285-1292

DOI: 10.2967/jnumed.111.088658

$\mathbf{S}$ for observing biologic pathways and cellular function in for observing biologic pathways and cellular function in

Received Jan. 31, 2011; revision accepted Apr. 20, 2011.

For correspondence or reprints contact: R. Glenn Wells, 40 Ruskin St., Ottawa, ON, K1Y 4W7 Canada.

E-mail: gwells@ottawaheart.ca

COPYRIGHT @ 2011 by the Society of Nuclear Medicine, Inc. vivo for medical diagnosis and research (1). Pinhole SPECT systems designed for preclinical imaging of mouse and rat models can provide images of tracer distribution with submillimeter resolution (2-4). Such small-animal SPECT systems are a valuable tool for researching disease progression and treatment efficacy in small-animal models of human diseases. Various rat models of cardiovascular diseases and modes of heart failure have been developed over the past few decades (5). With electrocardiogram (ECG) gating, high-resolution small-animal SPECT cameras can be used to measure aspects of cardiac function including myocardial perfusion, left ventricle (LV) volume, and ejection fraction in rats.

Before small-animal SPECT data can be used for the investigation of rat models of cardiac disease, a baseline of reference data along with an assessment of measurement reproducibility is a vital prerequisite. A reference database of LV perfusion, volume, and motion has been reported for mice (6), but we are not aware of similar data being available for rats.

Small-animal SPECT measurements are subject to various uncertainties. Some of these arise from the nature of SPECT itself, such as statistical noise. Other uncertainties are associated with the subject being imaged, for example, the challenge of maintaining a constant physiologic state in a live animal over an appreciable length of time. Finally, additional uncertainty may be introduced by the techniques used to analyze the images and extract relevant measurements. Understanding the magnitude of these uncertainties is essential any time small-animal SPECT is used to measure and compare cardiac parameters, such as when evaluating changes from disease progression, intervention, or the introduction of new technology or scanning techniques.

The test-retest reproducibility of single-pinhole clinical camera SPECT measurements of LV function in rats has been reported by Vanhove et al. (7). Measurements on healthy rats performed $1 \mathrm{wk}$ apart showed no significant differences in ejection fraction or LV volume. Modern small-animal SPECT systems now use multiple detectors 
and multiple pinholes to obtain cardiac SPECT studies in reasonable scan times with considerably reduced injected activity.

The objective of this study was to determine the intrasubject and intersubject reproducibility of serial measurements of perfusion, myocardial defect severity, LV volume, and ejection fraction, using ${ }^{99 \mathrm{~m}} \mathrm{Tc}$-tetrofosmin and a multiplexed multipinhole small-animal SPECT scanner in healthy rats and rats after myocardial infarction.

\section{MATERIALS AND METHODS}

\section{Scan Protocol}

Twenty male Sprague-Dawley rats were included in this study. Eight rats were given a myocardial infarction by surgical occlusion of the left anterior descending artery $4 \mathrm{wk}$ before the initial scan. The healthy rats $(n=8)$ were $12 \mathrm{wk}$ old at the start of the study, with a mean initial weight of $245 \pm 15 \mathrm{~g}$. The infarcted rats $(n=8)$ were $16 \mathrm{wk}$ old at the start of the study, with mean initial weight of $432 \pm 59 \mathrm{~g}$. The reference perfusion database was created using the data from the 8 healthy rats, plus an additional 4 healthy rats, also of the same strain, age, and sex. The 12 healthy rats had a mean initial weight of $254 \pm 18 \mathrm{~g}$. The study was approved by the Animal Care Ethics Committee of the University of Ottawa.

The rats were anesthetized with $2 \%-3 \%$ isoflurane and injected via the tail vein with $78 \pm 15 \mathrm{MBq}$ of ${ }^{99 \mathrm{~m}} \mathrm{Tc}$-tetrofosmin. All scans were performed with the rat supine, similar to the position used clinically.

All images were acquired with a nanoSPECT small-animal camera (Bioscan). This camera uses a multiplexed, multipinhole configuration with 4 detectors, allowing a full $360^{\circ}$ of projection data to be acquired by a $90^{\circ}$ rotation of the gantry. Translation of the rat through the bore of the scanner as the projections were acquired resulted in a helical scanning pattern. In total, 48 projections were acquired, equally spaced around the rat. The pinhole collimator in front of each detector had a grid of nine 2-mm focusing pinholes. Although this configuration resulted in some ambiguity at the detector due to overlapping projections from different pinholes, this issue was dealt with in the vendor-supplied software by an appropriately designed reconstruction algorithm (8).

The temperature of the rat was maintained by a heated Minerve animal bed (Bioscan) maintained between $34^{\circ} \mathrm{C}$ and $35^{\circ} \mathrm{C}$. To avoid interference from the probe, the temperature of the rat was not directly monitored during the scans. We have previously measured the relationship between the bed temperature and the rat body temperature. The rat body temperature was found to be stable at $35.4^{\circ} \mathrm{C} \pm 0.9^{\circ} \mathrm{C}$ for bed temperatures over a range of $33^{\circ} \mathrm{C}-36^{\circ} \mathrm{C}$.

A small-animal monitoring and gating system (model 1025T; SA Instruments) was used to monitor the breathing and heart rate of the rat. Breathing was detected during the scan by a pressuresensitive pad placed under the rat. Heart rate was monitored during the scan by 3 ECG leads. Initial scans were done with adhesive neonatal ECG electrodes attached to the paws; subcutaneous ECG leads were found to provide a more reliable ECG trace and were used for later scans. The rats were anesthetized during placement of the leads, and no discomfort arising from the use of subcutaneous leads was evident. The data were gated by dividing the R-R interval of the ECG into 8 segments of equal duration and assigning each count detected to a frame corresponding to its position in the cardiac cycle. The nominal R-R interval was set to an initial value, typically in the range 150-200 ms (equivalent to 300-400 bpm). Data collected during beats with an R-R interval that differed from the nominal interval by more than $9 \%$ were discarded. If the heart rate drifted during the acquisition, the nominal R-R interval was automatically adjusted up or down by the software.

Based on previous work done in our laboratory that measured the dependence of reproducibility on acquisition duration, a total scan duration of $30 \mathrm{~min}$ was chosen for all studies. Scans of the healthy rats were obtained in 10-min segments beginning 30, 40, $50,60,70$, and $80 \mathrm{~min}$ after tracer injection, and the projection data from the three 10-min time segments beginning at 50,60, and $70 \mathrm{~min}$ after injection were summed before reconstruction, resulting in a total of 7-12 million counts used for each reconstruction. Scans of the infarcted rats were obtained as a single 30min data acquisition commencing $30 \mathrm{~min}$ after injection, though the protocol was otherwise identical. Some of the early scans of the healthy rats were missing the data from the 30- and 40-min postinjection time frames. Because biodistribution of tetrofosmin is stable after $30 \mathrm{~min}$, we do not expect there to be an appreciable difference between scans started 30 min after injection and scans started $50 \mathrm{~min}$ after injection.

The healthy rats were scanned once per week for $5 \mathrm{wk}$. The infarcted rats were scanned twice per week, also for a total of 5 scans, over $15 \mathrm{~d}$ starting $4 \mathrm{wk}$ after infarction. The timing of the infarcted-rat scans after surgery was chosen to minimize changes due to further remodeling or healing during the course of the 5 imaging sessions. Previous work has shown that the infarct size in the rat stabilizes by approximately 3 wk after surgery (9).

Projection data were reconstructed using the vendor software supplied with the nanoSPECT system. The reconstruction parameters included a 3D gaussian filter between iterations, with a full width at half maximum of $0.63 \mathrm{~mm}$ ("high" smoothing), a voxel size of $0.6 \times 0.6 \times 0.6 \mathrm{~mm}$, and 24 iterations ( 8 iterations of 6 subsets, 8 iterations of 4 subsets, and 8 iterations of 3 subsets; "high" setting).

The reconstructed SPECT scans were analyzed with 4DMSPECT (Invia) to measure perfusion, ungated LV volume, LV ejection fraction, and the volumes of the LV at systole and diastole. To analyze a rat-sized heart using 4D-MSPECT software, the voxel dimensions were relabeled to be $6 \times 6 \times 6 \mathrm{~mm}$ to obtain an approximately human-sized heart. Within 4D-MSPECT, the heart was oriented and the valve plane specified by the user. For consistency, the location of the valve plane was determined by the location of the edge of the inferior wall of the myocardium in the horizontal short axis. From the ungated data, 4D-MSPECT calculated the ungated LV volumes and created a perfusion map for each scan. The perfusion map was normalized with respect to the point of maximum perfusion. Using a 17-segment cardiac model, the average perfusion (as a percentage of the maximum perfusion) was calculated for each segment. The LV ejection fraction and the volume of the LV at systole and diastole were calculated from the reconstruction of the cardiac gated SPECT data. To minimize interoperator variability in the calculated volumes and ejection fraction, all data were processed by a single user.

To correct for changes in rat weight as the rats grew over the course of the study, the measured volumes were normalized to the weight of the rat. In view of 2 papers $(10,11)$ that reported a linear 
relationship between body weight and ventricle volume in mammals, we scaled the LV volumes to an equivalent volume corresponding to the average weight of the rats in the group of interest. For interscan repeatability, we scaled each volume by the ratio of the average weight of the rat over the 5 scans to the weight of the rat at the time of the scan. Likewise, for intersubject repeatability, we corrected the volumes to the average weight of all 8 rats at that time point. Figure 1 shows the LV volumes measured for 1 of the rats before and after rescaling.

\section{Data Analysis}

Both the healthy-rat and the infarcted-rat studies comprised a total of 8 animals, each scanned 5 times, for a total of 40 sets of scan data per study. For the parameters calculated, intrasubject reproducibility was specified by the variation in an observed parameter when the same rat was scanned and analyzed repeatedly. Intersubject reproducibility was specified by the variation in a parameter measured in a large group of rats. To calculate the intrasubject reproducibility of a particular parameter, we grouped data by rat, calculated the SD of the parameter for each animal, and averaged the SD over all 8 rats. Intersubject reproducibility was calculated similarly, but with the data grouped by scan number instead of by rat. The SD of each parameter was calculated for each scan, and then the SD was averaged over all 5 scans. The significance of differences between reproducibility
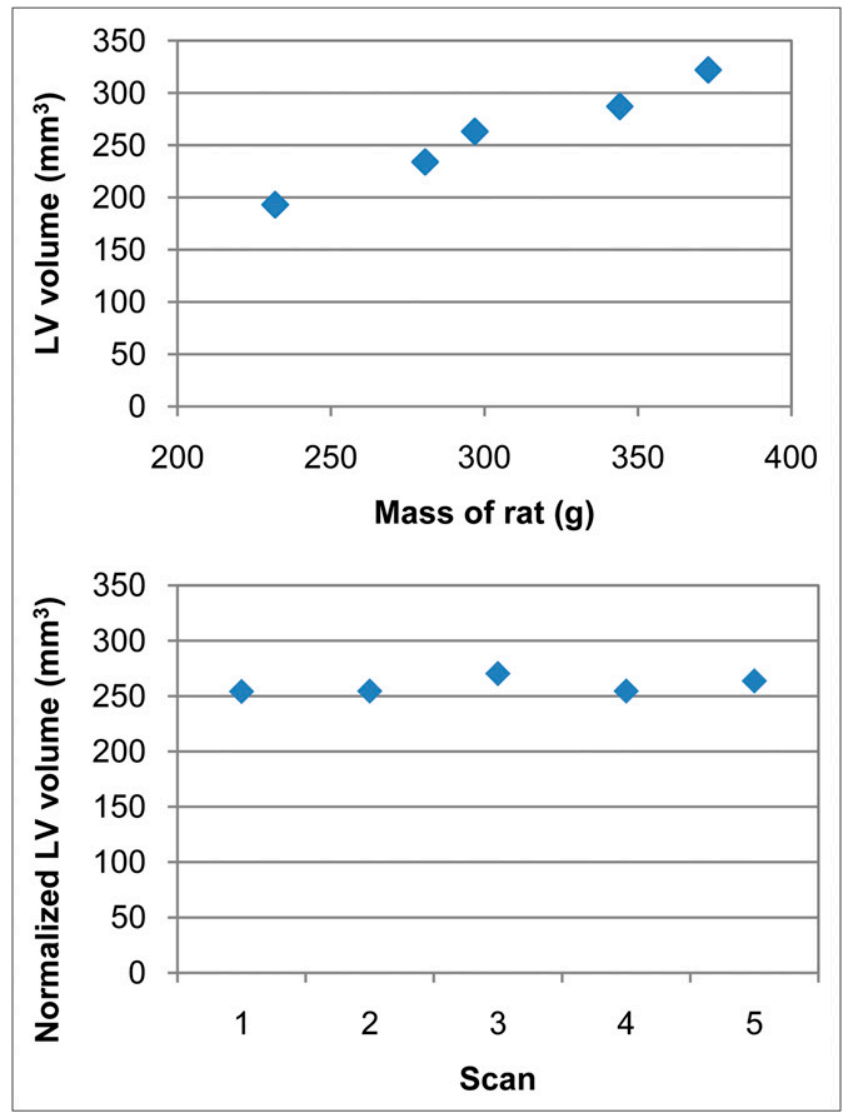

FIGURE 1. (Top) Volume of LV of rat 1 plotted against mass of rat. (Bottom) Volume of LV of rat 1 after volume normalization based on mass of rat at time of each scan. was assessed using the Welch $t$ test to compare the means of sample populations with unequal variances.

Perfusion. A reference database of myocardial perfusion was created by calculating the average and SD of the perfusion of each segment of the myocardium across all scans. The dataset used to create the reference database also contained the 8 rats for which the functional data were analyzed and an additional 4 male Sprague-Dawley rats for which we had perfusion data, but no functional data, for a total of 12 rats (60 total scans).

The interscan and intersubject reproducibilities were calculated for all 17 segments. For the infarcted rats, we also calculated a $z$ score for each segment. The $z$ score was the difference between the measured perfusion in the segment and the corresponding segment in the reference database, divided by the SD for that segment. We also calculated, as an overall measure of myocardial damage, a combined $z$ score for each scan, defined as the sum of the $z$ scores for all segments with a $z$ score less than or equal to -2.0 . We also measured the reproducibility of the perfusion measurements for the unaffected segments of the infarcted rat heart to evaluate the applicability of the healthy-rat database to infarctedrat models. We chose only those segments that had a normalized perfusion greater than $75 \%$ in all the rats scanned and compared the measured perfusion in those segments with the reference database.

Functional Measurements. To factor out the normal growth of the rat from the interscan and intersubject reproducibility of the volume measurements (end-diastolic and end-systolic LV volumes), all volumes were normalized to the weight of the rat. For interscan reproducibility calculations, the volumes were normalized to the average weight of each rat over the course of the study. For intersubject reproducibility, the volumes were normalized to the average weight of the rats in each scan. The reproducibility of the ejection fraction was also calculated, but in this case no normalization was required. The reproducibilities of the end-systolic volume, end-diastolic volume, and ejection fraction were determined for the healthy rats and the infarcted rats.

\section{RESULTS}

\section{Scanning}

At the time of the first scan, the average weight of the 8 healthy rats was $245 \pm 15 \mathrm{~g}$. At the time of the final scan, their average weight was $383 \pm 41 \mathrm{~g}$. The 8 infarcted rats began with weights of $432 \pm 59 \mathrm{~g}$ and had weights of $475 \pm 51 \mathrm{~g}$ by the final scan.

During scan acquisition, the healthy rats had an average heart rate of $320 \pm 30 \mathrm{bpm}$ and a respiration rate of $60 \pm$ $9 \mathrm{breaths} / \mathrm{min}$. The infarcted rats had heart and respiration rates of $337 \pm 47 \mathrm{bpm}$ and $58 \pm 11$ breaths/min, respectively.

The total number of counts used to reconstruct the images was 7-12 million for the ungated images and about 1 million per phase for the gated images. On average, badbeat rejection caused a loss of $15 \%$ of the counts in the gated data, compared with the ungated data.

\section{Perfusion}

Reproducibility of Measurements and Creation of Reference Database. A reference database created from the scans 


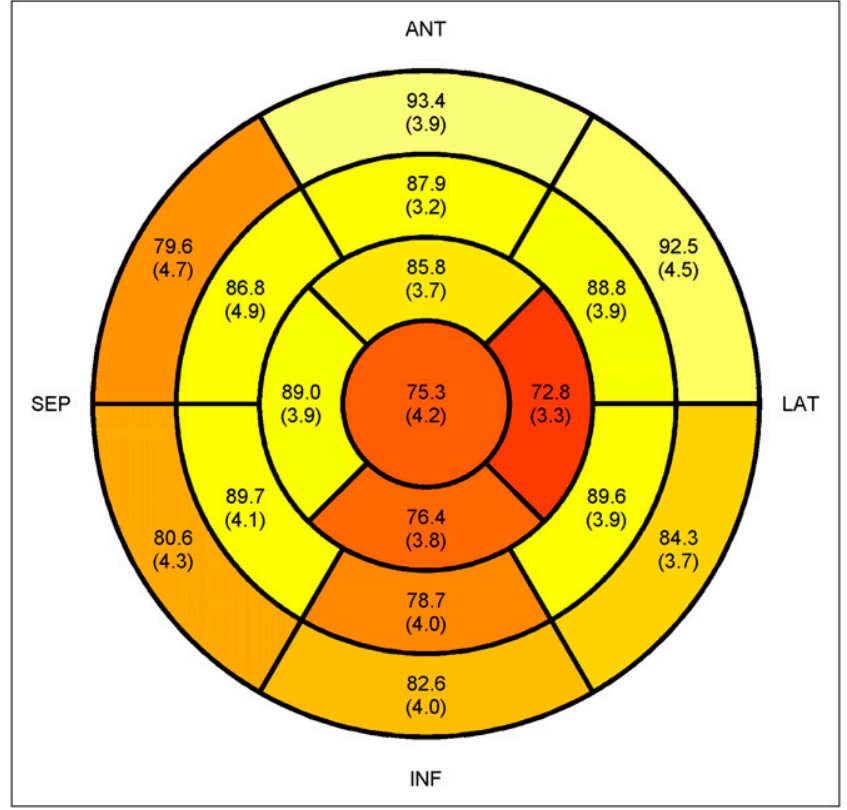

FIGURE 2. Reference database showing average perfusion and $\mathrm{SD}$ of average perfusion for each segment of myocardium. ANT = anterior; INF = inferior; LAT = lateral; SEP = septal.

of the healthy rats is shown in Figure 2 and provides the average perfusion and SD (over all scans) for each segment of the myocardium. Average segment perfusion ranged from $72.8 \%$ to $93.4 \%$, with SDs between $3.2 \%$ and $5.5 \%$. A region of comparatively low perfusion in the lateral wall near the apex was observed in all rats and is reflected in the reference database. The reason for this observation is unclear. An apparent reduction in uptake could be caused by partial-volume effects, but the myocardium did not appear thinner near the apex when dissected. The lowperfusion region has, however, been reproducible across all the rats we have scanned to date.

The minimum, maximum, and average of the segment perfusion reproducibilities are listed in Table 1 for the healthy rats and in Table 2 for the infarcted rats. The average interscan reproducibility of segment perfusion was $2.8 \%$, and the average intersubject reproducibility was $4.0 \%$. The reproducibility of the segment perfusion

TABLE 1

Reproducibility of Cardiac Perfusion Measurements in Healthy Rats

\begin{tabular}{ccc}
\hline \multicolumn{1}{c}{ Parameter } & Mean perfusion & Reproducibility \\
\hline Interscan & & \\
Least variable segment & $86.8 \%$ & $2.4 \%$ (segment 8$)$ \\
Most variable segment & $80.6 \%$ & $3.6 \%$ (segment 3) \\
Average & $84.3 \%$ & $2.8 \%$ \\
Intersubject & & \\
Least variable segment & $87.9 \%$ & $3.2 \%$ (segment 7) \\
Most variable segment & $86.8 \%$ & $5.0 \%$ (segment 8) \\
Average & $84.3 \%$ & $4.0 \%$ \\
\hline
\end{tabular}

TABLE 2

Reproducibility of Cardiac Perfusion Measurements in Infarcted Rats

\begin{tabular}{ccc}
\hline \multicolumn{1}{c}{ Parameter } & $\begin{array}{c}\text { Mean } \\
\text { perfusion }\end{array}$ & Reproducibility \\
\hline Interscan & & \\
Least variable segment & $21.4 \%$ & $3.1 \%$ (segment 16) \\
Most variable segment & $76.5 \%$ & $7.1 \%$ (segment 6) \\
Average & $63.1 \%$ & $5.3 \%$ \\
Intersubject & & \\
Least variable segment & $82.7 \%$ & $5.0 \%$ (segment 8) \\
Most variable segment & $58.5 \%$ & $26.9 \%$ (segment 11) \\
Average & $63.1 \%$ & $14.4 \%$
\end{tabular}

measurements was worse for the infarcted rats: $5.3 \%$ and $14.4 \%$ for interscan and intersubject reproducibility, respectively.

If the segments are divided into uninfarcted segments $(z$ score $>-2.0)$, moderately infarcted segments $(z$ score between -2.0 and -4.0$)$, and severely infarcted segments $(z$ score $<-4.0)$, the average interscan segment perfusion reproducibilities were $3.6 \%, 5.2 \%$, and $7.2 \%$, respectively.

The validity of using the reference database for evaluation of infarcted rats was assessed by comparing, with the reference database, only those segments in the infarcted-rat scans that had an average normalized perfusion exceeding $75 \%$ in all 8 rats. In the rats studied, 3 segments, all in the septal wall of the heart, met this criterion. These segments had average perfusions of $86.0 \% \pm 6.6 \%, 82.7 \% \pm 5.3 \%$, and $86.5 \% \pm 5.4 \%$. The corresponding segments in the reference database were $89.7 \% \pm 4.1 \%, 86.8 \% \pm 4.9 \%$, and $89.0 \% \pm 3.9 \%$, respectively.

Reproducibility of Infarct Severity Measurements. Figure 3 shows the $z$ scores for 1 of the rats. A combined $z$ score indicating the overall size and severity of the infarct was calculated for each rat (Table 3). The average score for each rat ranged from -54 for the least severely infarcted animal to -143 for the most severely infarcted. For individual rats, the average interscan SD of the combined $z$ score was 8 . The average SD of the intersubject combined $z$ scores was 28 .

\section{Functional Measurements}

Reproducibility of Systolic and Diastolic Volumes. The mean values and the reproducibilities of the systolic and diastolic LV volumes for the healthy and infarcted rats are shown in Tables 4 and 5, respectively. For both, the intersubject variation was significantly larger than the interscan variation (end-systolic volume, $P=0.003$; end-diastolic volume, $P=0.006$ ). Interscan reproducibility was $4 \%-$ $7 \%$ of the total volume, and intersubject reproducibility was $9 \%-12 \%$ of total ventricle volume for the healthy rats.

Table 5 lists the corresponding reproducibilities for the infarcted rats. In the infarcted rats, the intersubject varia- 


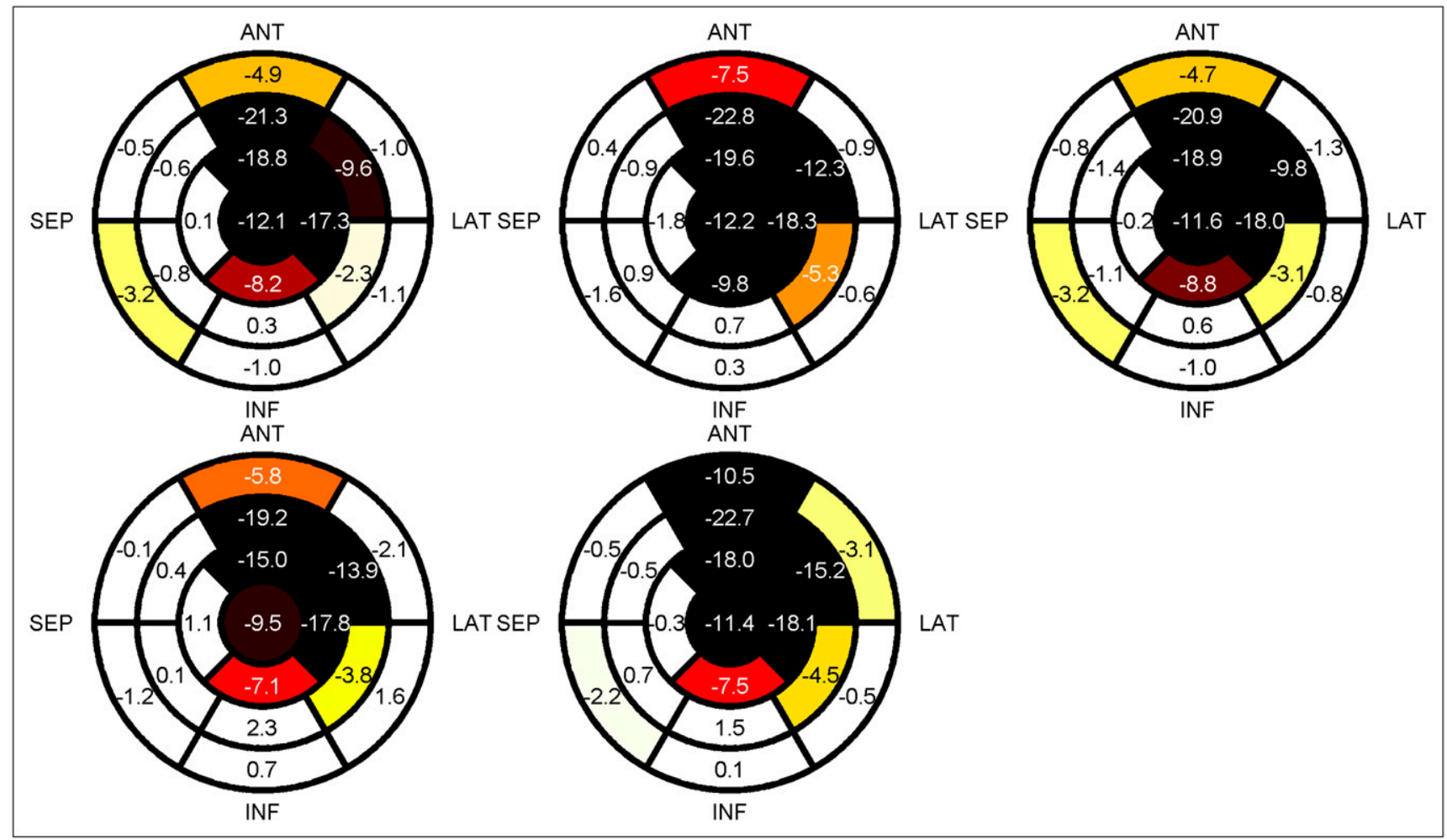

FIGURE 3. $z$ scores for all 5 scans of 1 rat. ANT = anterior; INF $=$ inferior; LAT $=$ lateral; SEP $=$ septal.

tions of the systolic and diastolic volumes were much larger than interscan variances $(P<0.001$ in both cases).

For the infarcted rats, the interscan variances were larger in absolute terms than those for healthy rats, but the average volume was also larger because the infarcted rats were older and because of myocardial remodeling after the infarction. Expressed as a percentage of average ventricle volume, the interscan reproducibility was $6 \%-8 \%$, marginally worse than the relative interscan reproducibility for the healthy rats.

Intersubject reproducibility was much worse for the infarcted rats: $15 \%-26 \%$ of the average volume of the LV.

Reproducibility of Ejection Fraction. Ejection fraction reproducibility for the healthy rats is also included in Tables 4 and 5. Unlike for the LV volumes, there was no significant difference between the reproducibilities of $3.1 \%$

TABLE 3

Average Combined $z$ Scores for 5 Scans of 8 Infarcted Rats

\begin{tabular}{lc}
\hline Rat & Combined $z$ score \\
\hline 1 & $-88 \pm 22$ \\
2 & $-115 \pm 7$ \\
3 & $-102 \pm 8$ \\
4 & $-84 \pm 8$ \\
5 & $-54 \pm 5$ \\
6 & $-143 \pm 5$ \\
7 & $-84 \pm 8$ \\
8 & $-82 \pm 4$ \\
\hline
\end{tabular}

and $3.3 \%$ for the intersubject and interscan ejection fraction measurements, respectively $(P=0.86)$. The interscan ejection fraction reproducibility for the infarcted rats, at 3.6\%, was not significantly different from the reproducibility for the healthy rats $(P=0.64)$. The intersubject reproducibility of $8.1 \%$ was significantly worse than the interscan reproducibility for the infarcted rats $(P=0.01)$.

\section{DISCUSSION}

In every case, reproducibility, as measured by the SD of repeated measurements, was worse for intersubject meas-

TABLE 4

Reproducibility of Functional Measurements in Healthy Rats

\begin{tabular}{ccc}
\hline Parameter & Mean & Reproducibility \\
\hline Interscan $(n=8)$ & & \\
Ejection fraction & $57 \%$ & $3.1 \% \pm 1.6 \%$ \\
Diastolic volume & $470 \mathrm{~mm}^{3}$ & $20 \pm 7 \mathrm{~mm}^{3}$ \\
Systolic volume & $203 \mathrm{~mm}^{3}$ & $13 \pm 6 \mathrm{~mm}^{3}$ \\
Intersubject $(n=5)$ & & \\
Ejection fraction & $57 \%$ & $3.3 \% \pm 1.5 \%$ \\
Diastolic volume & $470 \mathrm{~mm}^{3}$ & $44 \pm 16 \mathrm{~mm}^{3}$ \\
Systolic volume & $204 \mathrm{~mm}^{3}$ & $24 \pm 5 \mathrm{~mm}^{3}$
\end{tabular}

Volumes are after normalization. Because interscan data are normalized to average weight of each rat, and intersubject data are normalized to average weight of all rats for each scan, the means differ slightly. 
TABLE 5

Reproducibility of Functional Measurements in Infarcted Rats

\begin{tabular}{ccc}
\hline Parameter & Mean & Reproducibility \\
\hline Interscan $(n=8)$ & & \\
Ejection fraction & $45 \%$ & $3.6 \% \pm 2.4 \%$ \\
Diastolic volume & $834 \mathrm{~mm}^{3}$ & $49 \pm 12 \mathrm{~mm}^{3}$ \\
Systolic volume & $462 \mathrm{~mm}^{3}$ & $37 \pm 17 \mathrm{~mm}^{3}$ \\
Intersubject $(n=5)$ & & \\
Ejection fraction & $45 \%$ & $8.1 \% \pm 2.4 \%$ \\
Diastolic volume & $832 \mathrm{~mm}^{3}$ & $126 \pm 20 \mathrm{~mm}^{3}$ \\
Systolic volume & $462 \mathrm{~mm}^{3}$ & $121 \pm 14 \mathrm{~mm}^{3}$
\end{tabular}

Volumes are after normalization. Because interscan data are normalized to average weight of each rat, and intersubject data are normalized to average weight of all rats for each scan, the means differ slightly.

urements than for interscan measurements, underscoring the benefit of using noninvasive methods such as SPECT for longitudinal studies. The benefits of interscan over intersubject comparison are even more evident in infarcted rats, for which the underlying physiologic variance between subjects is much greater than in healthy rats. This variance reflects the difficulty of creating a consistent infarction across multiple animals using surgical methods and is most evident in the large intersubject variation in $z$ scores and ejection fraction in the infarcted group, compared with the interscan variation of the same parameters. As was visibly evident on the scans, the region of infarcted tissue was much larger in some rats than in others. Severely infarcted myocardia are also more difficult to align consistently during analysis, because the remaining viable tissue may not provide a clear image of the rotation and extent of the heart. The greater uncertainty in the position of severely infarcted segments may contribute to the poorer reproducibility of perfusion measurements in more severely infarcted segments. For infarcted rats, the interscan reproducibility of segment perfusion decreases as the severity of the perfusion defect increases. Interscan reproducibility of uninfarcted segments is no worse than in healthy rats. Furthermore, measurements of perfusion in the uninfarcted segments of infarcted rats were consistent with the same measurements made in healthy rats, summarized in the reference database. The interscan SD of relative perfusion was about twice as large in severely infarcted segments as in uninfarcted segments.

At this time, data on the reproducibility of cardiac SPECT studies in small animals are limited. Moreover, direct comparison between our work and that of Vanhove et al. (7), who reported reproducibility in terms of average pairwise differences between LV volumes measured 1 wk apart, is difficult. The magnitudes of the pairwise differences observed by Vanhove et al. are consistent with variability observed in our study. Comparison with similar studies on human subjects is also challenging, because few studies have investigated the repeatability of measurements over repeated imaging in humans. Xu et al. (12) evaluated the reproducibility of functional measurements in humans and reported a $95 \%$ confidence interval $(-10.1 \%$, $10.2 \%$ ) for test-retest reproducibility of ejection fraction measurements in humans. The interscan reproducibility of ejection fraction observed in rats, $3.3 \%$, equates to an expected $95 \%$ confidence interval for repeated measurements of $\pm 9 \%$, consistent with the results of the human study.

Some of the variation in the measured values of segment perfusion, ejection fraction, and LV volume reflects actual differences in physiology; other variations arise from inherent uncertainties in the procedure. Over time, gradual changes in the physiology are expected. Despite the effort to maintain a consistent physiologic state for each scan, some confounding factors, such as the level of anesthetic, are unavoidable. Minor differences in physiologic state are reflected in the ranges of heart and breathing rates between different scans and different animals. Intersubject variations were minimized by using animals of the same age, sex, and strain, but differences between individual animals still exist and contribute to the variations observed in the measured parameters. In clinical imaging, attenuation causes sexspecific artifacts and requires the use of sex-specific databases. With rats, the impact of attenuation is greatly reduced because of the small size of the subject, and we expect the reproducibility to be similar for female rats of the same strain. Nevertheless, these results are specific to male rats, and the effect of sex was not evaluated.

Variations in the scanning and analysis process unrelated to variations between rats also influence repeatability. Variations would include exact placement of the animal and noise in the imaging process. Image analysis introduces additional operator-dependant factors in the manual rotation and alignment of the reconstructed image and the placement of the valve plane. In this study, all images were processed by a single operator to minimize interoperator variation. The use of multiple operators for data analysis would increase the measured uncertainties. However, the interobserver variability in small-animal myocardial perfusion SPECT reported by Vanhove et al. (7) is comparatively small and not significantly different from the intraobserver variability.

Increasing the number of frames used for ECG gating may improve the absolute accuracy of LV volume measurements, particularly at systole, and the accuracy of the ejection fraction measurement. However, without increasing the scan duration, the additional subdivision of data into a greater number of time divisions would increase the noise in each reconstructed frame, potentially degrading accuracy and reproducibility, though to what degree is uncertain.

One major advantage of using SPECT to evaluate changes in a living subject is the ability to use an animal as its own control. We have demonstrated that the interscan 
reproducibility of LV volume measurements is significantly better than the intersubject reproducibility. Relative to studies that rely entirely on intersubject comparison, as when subjects must be sacrificed for analysis, SPECT permits a significant increase in statistical power with the same sample size or equivalent statistical power with a smaller sample size. For example, consider a study of ejection fraction in infarcted rats wherein we want to detect a change of 5\% with an $\alpha$ of 0.05 and a power of 0.95 . Using 2 independent groups of rats, each with a SD of $8.1 \%$ for ejection fraction when measured using small-animal SPECT, a total sample size of 140 rats would be required. If the study were done as a serial study with each animal compared against itself (before and after treatment), the SD of the difference is reduced to $5.1 \%$ and a paired $t$ test requires only 16 animals, a 9-fold reduction.

The linear model we used to correct for normal growth exhibited reasonable conformity to the growth trends observed in the healthy rats we studied, which had weights ranging from 200 to $450 \mathrm{~g}$. The disease state of the animal model may limit the applicability of this linear correction. Although we also used the same assumed weight-LV volume relationship to normalize the data in the infarcted rats included in this study, the physiologic consequences of an induced myocardial infarction may change the relationship between the normal heart volume and the weight of the rat, undermining the validity of this assumption. Any error in this assumption was mitigated, however, by the shorter duration of the myocardial infarction study. Because the infarcted rats were scanned over a shorter period, they grew comparatively less during the study and consequently required much less normalization than did the healthy rats.

To measure perfusion, LV volume, and ejection fraction, we used 4D-MSPECT. Though 4D-MSPECT has been validated for human cardiac measurements (13), certain assumptions about the shape and thickness of a human myocardium may not be entirely appropriate for a rat heart. In particular, 4D-MSPECT will not process a rat-sized heart. As noted in the "Materials and Methods" section, we scaled the apparent voxel size in the reconstructed heart data by a factor of 10 . The LV volume calculated by the software changed substantially with changes to the apparent voxel size. Within the range of $8-11$ for the scaling factor, the volumes were constant and consistent with measurements of healthy-rat end-diastolic volume by others (7,14-16). This method was further validated using a rat myocardium phantom, scanned with the same protocol as the rats in the study. The volume calculated by 4DMSPECT was approximately $20 \%$ larger than the true volume, and we believe this difference to be a consequence of the software's rounding off of the flat bottom of the phantom to make it more heartlike, increasing the apparent volume. These uncertainties in the absolute accuracy of the volume measurement are not expected to have a significant impact on the conclusions of this paper. Further work may be warranted to validate the measurement on rat heart vol- umes using software designed for clinical use. (Invia has expressed an intention of extending the 4D-MSPECT software to handle rat hearts, but the software was not yet available at the time of this study.)

\section{CONCLUSION}

We have created a reference database for myocardial perfusion of male Sprague-Dawley rats imaged with 99m Tc-tetofosmin. We have also measured the repeatability of perfusion measurements in healthy rats and in rats with a coronary artery ligation-induced myocardial infarction. Finally, we have measured the reproducibility of nanoSPECT functional measurements of LV diastolic volume, systolic volume, and ejection fraction.

\section{DISCLOSURE STATEMENT}

The costs of publication of this article were defrayed in part by the payment of page charges. Therefore, and solely to indicate this fact, this article is hereby marked "advertisement" in accordance with 18 USC section 1734.

\section{ACKNOWLEDGMENTS}

We thank Myra Kordos and Julia Lockwood for assisting with the animal imaging studies. We also thank Monir Ahmad for performing the myocardial infarction surgeries and Esta Nel for assisting with the analysis of rat and bed temperature. The research was supported by grant NA6374 from the Heart and Stroke Foundation of Ontario, operating grant FRN: MOP-13182 from the Canadian Institutes of Health Research, the Ontario Research Fund (RE-02-038), a PGS D scholarship from the National Sciences and Engineering Research Council of Canada, an endowed chair supported by Pfizer Canada, the University of Ottawa Heart Institute Foundation, the Canadian Institutes of Health Research, and the Vered Chair in Cardiology at the University of Ottawa Heart Institute. No other potential conflict of interest relevant to this article was reported.

\section{REFERENCES}

1. Weissleder R, Mahmood U. Molecular imaging. Radiology. 2001;219:316-333.

2. Meikle SR, Kench P, Kassiou M, Banati RB. Small animal SPECT and its place in the matrix of molecular imaging technologies. Phys Med Biol. 2005;50:R45-R61.

3. Franc BL, Acton PD, Mari C, Hasegawa BH. Small-animal SPECT and SPECT/ CT: important tools for preclinical investigation. J Nucl Med. 2008;49:1651-1663.

4. Nuyts J, Vunckx K, Defrise M, Vanhove C. Small animal imaging with multipinhole SPECT. Methods. 2009;48:83-91.

5. Hasenfuss G. Animal models of human cardiovascular disease, heart failure, and hypertrophy. Cardiovasc Res. 1998;38:60-76.

6. Constantinesco A, Choquet P, Monassier L, Israel-Jost V, Mertz L. Assessment of left ventricular perfusion, volumes, and motion in mice using pinhole gated SPECT. J Nucl Med. 2005;46:1005-1011.

7. Vanhove C, Lahoutte T, Defrise M, Bossuyt A, Franken PR. Reproducibility of left ventricular volume and ejection fraction measurements in rat using pinhole gated SPECT. Eur J Nucl Med Mol Imaging. 2005;32:211-220.

8. Schramm NU, Ebel G, Engeland U, Schurrat T, Béhé M, Behr TM. Highresolution SPECT using multipinhole collimation. IEEE Trans Nucl Sci. 2003;50:315-320.

9. Fishbein MC, Maclean D, Maroko PR. Experimental myocardial infarction in the rat. Am J Pathol. 1978;90:57-70. 
10. Holt JP, Rhode EA, Kines H. Ventricular volumes and body weight in mammals. Am J Physiol. 1968;215:704-715.

11. Dawson TH. Similitude in the cardiovascular system of mammals. $J$ Exp Biol. 2001;204:395-407.

12. Xu Y, Hayes S, Ali I, et al. Automatic and visual reproducibility of perfusion and function measurements for myocardial perfusion SPECT. J Nucl Cardiol. 2010; 17:1050-1057.

13. Nakajima K, Higuchi T, Taki J, Kawano M, Tonami N. Accuracy of ventricular volume and ejection fraction measured by gated myocardial SPECT: comparison of 4 software programs. J Nucl Med. 2001;42:1571-1578.
14. Wise RG, Huang CLH, Gresham GA, Al-Shafei AIM, Carpenter TA, Hall LD. Magnetic resonance imaging analysis of left ventricular function in normal and spontaneously hypertensive rats. J Physiol. 1998;513:873887.

15. Nahrendorf M, Wiesmann F, Hiller KH, et al. Serial cine-magnetic resonance imaging of left ventricular remodeling after myocardial infarction in rats. J Magn Reson Imaging. 2001;14:547-555.

16. Croteau E, Bénard F, Cadorette J, et al. Quantitative gated PET for the assessment of left ventricular function in small animals. J Nucl Med. 2003;44:16551661 . 